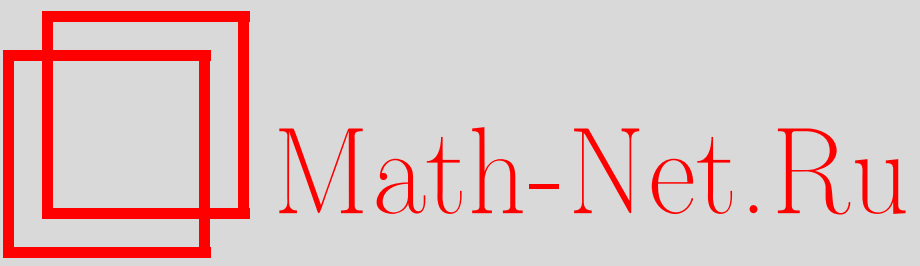

X. K. Ишкин, X. Х. Муртазин, О квантовом дефекте для оператора Дирака с неаналитическим потенциалом, ТМФ, 2000, том 125, номер 3, 444-452

DOI: https://doi.org/10.4213/tmf678

Использование Общероссийского математического портала Math-Net.Ru подразумевает, что вы прочитали и согласны с пользовательским соглашением

http://www.mathnet.ru/rus/agreement

Параметры загрузки:

IP : 3.85 .183 .62

26 апреля 2023 г., 09:58:23 


\section{О КВАНТОВОМ ДЕФЕКТЕ ДЛЯ ОПЕРАТОРА ДИРАКА С НЕАНАЛИТИЧЕСКИМ ПОТЕНЦИАЛОМ}

Вычислены квантовые дефекты непрерывного и дискретного спектров радиального уравнения Дирака с потенциалом $V(r)=-A / r+q(r)$, где $A>0, \quad \int_{0}^{\infty}|q| \times$ $(1+\sqrt{r}) d r<\infty$.

\section{1. ВВЕДЕНИЕ}

Понятие "квантового дефекта" было впервые введено в известной монографиии Зоммерфельда [1] для характеристики дискретного спектра некоторого возмущения оператора Шредингера атома водорода. С математической точки зрения "квантовый дефект" представляет собой величину $\delta$, на которую нужно сдвинуть главное квантовое число $n$ в асимптотической формуле для собственных чисел, чтобы получить первую поправку от возмушения. Это означает, что если $\mu_{n}$ и $\lambda_{n}-$ собственные числа соответственно возмущенного и невозмушенного операторов и если $\lambda_{n} \sim F(n), n \rightarrow \infty$, где $F(n)$ - известное выражение, то $\mu_{n} \sim F(n+\delta), n \rightarrow \infty$. Такой вид вклада от регулярного возмущения характерен для дифференциальных операторов с дискретным спектром, имеюшим конечную предельную точку. При этом величина $\delta$ может быть найдена в явном виде только для некоторых специальных потенциалов, а в общей ситуации она выражается в терминах решения уравнения при предельном значении спектрального параметра.

В работе [2] найдены значения квантового дефекта для непрерывного и дискретного спектров радиального уравнения Дирака с потенциалом

$$
V(r)=-\frac{A}{r}+q(r)
$$

где $A$ - положительное число, $q(r)$ - вешественная функция, удовлетворяющая следующим условиям:

1) $q(r)$ аналитична в области $\operatorname{Re} r>0$ и непрерывна при $\operatorname{Re} r \geqslant 0$, кроме, быть может, точки $r=0$;

2) имеет место неравенство

$$
\int_{0}^{\infty} e^{L \sqrt{t}} \hat{q}(t) d t<\infty, \quad \forall L>0
$$

*Башкирский государственный университет, Уфа, Россия. E-mail: ishkinhk@bashedu.ru; murtazin@mail.ru 
где

$$
\hat{q}(t)=\sup _{|\beta| \leqslant \frac{\pi}{2}}|q(t \exp i \beta)|
$$

Метод работы [2] специфичен для потенциалов указанного вида и существенно использует тот факт, что некоторая функция - аналог функции Йоста - может быть аналитически продолжена на нефизический лист.

В данной работе предлагается метод, который позволяет вычислить квантовый дефект оператора Дирака с потенциалом (1) в предположении выполнения лишь условия

$$
\int_{0}^{\infty}|q(r)|(1+\sqrt{r}) d r<\infty
$$

Кроме того, наша методика применима к более широкому, чем (1), классу потенциалов, содержащему, например, потенциалы $V$, дважды дифференцируемые и такие, что

$$
a_{k}\left((r+1)^{-\alpha}\right)^{(k)}<V^{(k)}(r)<A_{k}\left((r+1)^{-\alpha}\right)^{(k)}, \quad \alpha>0, \quad k=0,1,2 .
$$

\section{2. КВАНТОВЫЙ ДЕФЕКТ ДИСКРЕТНОГО СПЕКТРА}

Рассмотрим спектральную задачу для радиального уравнения Дирака

$$
\left(J \frac{d}{d r}+Q(r)\right) y=\lambda y, \quad y \in L^{2}(0, \infty) .
$$

Здесь

$$
J=\left(\begin{array}{cc}
0 & -1 \\
1 & 0
\end{array}\right), \quad Q(r)=\left(\begin{array}{cc}
V(r)+m & k / r \\
k / r & V(r)-m
\end{array}\right), \quad k>0, \quad m>0,
$$

$V(r)$ имеет вид (1), где $0<A<k$, функция $q(r)$ удовлетворяет условию $(2)$.

Известно, что дискретный спектр задачи (3) расположен внутри интервала $(-m, m)$ с единственной предельной точкой $m$. Пусть $\lambda_{n}$ - собственные числа уравнения $(3)$, пронумерованные в порядке возрастания.

ТЕОрема 1. Пусть q удовлетворяет условию (2) и $\alpha=\sqrt{k^{2}-A^{2}} \geqslant 1 / 2$. Тогда

$$
\lambda_{n}=m\left(1-\frac{A^{2}}{2(n+\delta+\alpha)}\right)+o\left(n^{-1}\right), \quad n \rightarrow \infty
$$

где $\delta=\beta / \pi$ и угол $\beta$ определяется равенствами (9)-(13) (см. ниже).

Число $\delta$ называется квантовым дефектом дискретного спектра.

1. Решение уравнения Дирака около нуля. В этом пункте мы построим квадратично-суммируемое в нуле решение уравнения (3), которое имеет известную асимптотику при $\lambda \rightarrow m$ равномерно по $r \in\left[0,|m-\lambda|^{-\delta}\right]$, где $0<\delta<2 / 3-$ постоянная (лемма 2). Перепишем (3) в виде

$$
y^{\prime}+\left(A_{0}-(z+q) J\right) y=0
$$


где

Введем обозначения

$$
A_{0}=\left(\begin{array}{cc}
k / r & -A / r-2 m \\
A / r & -k / r
\end{array}\right), \quad z=m-\lambda .
$$

$$
\begin{gathered}
T=\left(\begin{array}{cc}
1 & 1 \\
t & t^{-1}
\end{array}\right), \quad t=\frac{k+\alpha}{A} \\
B=B(r, z)=T^{-1}\left(A_{0}-z J\right) T, \quad R=-q T^{-1} J T .
\end{gathered}
$$

Тогда подстановка $y=T v$ приводит уравнение (4) к виду

$$
v^{\prime}+(B+R) v=0
$$

Сначала мы построим фундаментальную матрицу решений (ФМР) “предельного” уравнения

$$
v^{\prime}+\left(B_{0}+R\right) v=0,
$$

где $B_{0}=B(r, 0)$. Пусть $V_{0}-\Phi$ МР невозмушенного уравнения

$$
v^{\prime}+B_{0} v=0
$$

Легко проверить, что в качестве $V_{0}$ можно взять матрицу

$$
V_{0}=W^{\prime}-B_{0} W
$$

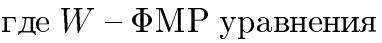

$$
w^{\prime \prime}-\left(B_{0}^{\prime}+B_{0}^{2}\right) w=0
$$

Имеем

следовательно,

$$
B_{0}^{\prime}+B_{0}^{2}=\operatorname{diag}\left(b_{1}, b_{2}\right), \quad b_{1,2}=-\frac{2 A m}{r}+\alpha(\alpha \pm 1) r^{-2},
$$

$$
\begin{gathered}
W=\operatorname{diag}\left(w_{1}, w_{2}\right), \\
w_{1}=\sqrt{r} J_{2 \alpha+1}(\sqrt{8 A m r}), \quad w_{2}=-t^{2} \sqrt{r} Y_{2 \alpha+1}(\sqrt{8 A m r}),
\end{gathered}
$$

где $J_{\nu}, Y_{\nu}-$ функции Бесселя.

Выпишем асимптотические формулы для $V_{0}$, которые нам понадобятся в дальнейшем,

$$
\begin{aligned}
V_{0}(r) & =\operatorname{diag}\left(a_{1}, a_{2}\right)(I+O(r)) \operatorname{diag}\left(r^{\alpha}, r^{-\alpha}\right), \quad r \rightarrow 0, \\
a_{1} & =(2 A m)^{\alpha+\frac{1}{2}}(\Gamma(2 \alpha+1))^{-1}, \quad a_{2}=-\frac{A m}{\pi \alpha} \frac{t^{2}}{a_{1}}, \\
V_{0}(r) & =T_{0} M_{1}(r)\left(\widehat{V}_{0}(r)+O\left(r^{-\frac{1}{2}}\right)\right), \quad r \rightarrow \infty, \\
T_{0} & =\frac{1}{\sqrt{\pi}}\left(\begin{array}{cc}
1 & -A m / \alpha \\
0 & (A m / \alpha) t^{2}
\end{array}\right), \quad M_{1}=\operatorname{diag}\left(\left(\frac{2 A m}{r}\right)^{\frac{1}{4}},\left(\frac{2 A m}{r}\right)^{-\frac{1}{4}}\right), \\
\widehat{V}_{0}(r) & =\left(\begin{array}{cc}
-\sin P_{1} & \cos P_{1} \\
\cos P_{1} & \sin P_{1}
\end{array}\right), \quad P_{1}=\sqrt{8 A m r}-\pi \alpha-\frac{3 \pi}{4} .
\end{aligned}
$$

Пусть $\Psi(r)$ - решение интегрального уравнения

$$
\Psi(r)=V_{01}(r)-\int_{0}^{r} V_{0}(r) V_{0}^{-1}(t) R(t) \Psi(t) d t
$$

где $V_{01}(r)$ - первый столбец матрицы $V_{0}(r)$. Ясно, что $\Psi(r)$ удовлетворяет уравнению (6). 
Лемма 1. Если выполнено условие (2), то

$$
\Psi(r) \sim \begin{cases}V_{01}(r), & r \rightarrow 0, \\ O\left(r^{\frac{1}{4}}\right), & r \rightarrow \infty .\end{cases}
$$

ДОкАЗАТЕЛЬСтво. Введем функцию

$$
\Omega(r)= \begin{cases}r^{-\alpha}, & 0<r \leqslant 1 \\ r^{-\frac{1}{4}}, & r>1\end{cases}
$$

и положим $\widetilde{\Psi}=\Omega \Psi, \widetilde{V}_{01}=\Omega V_{01}$. Из $(7)$ и (8) следует, что $\left|\widetilde{V}_{01}\right| \leqslant C_{0}, r>0 . \widetilde{\Psi}$ удовлетворяет уравнению

$$
\widetilde{\Psi}=\widetilde{V}_{01}-\int_{0}^{r}(\sqrt{t}+1) q(t) K(r, t) \widetilde{\Psi} d t
$$

где $|K(r, t)| \leqslant C, r, t>0$. Таким образом,

$$
|\widetilde{\Psi}| \leqslant C_{0} \exp \left(C \int_{0}^{r}(\sqrt{t}+1)|q(t)| d t\right),
$$

откуда и следует утверждение леммы.

Из асимптотических формул (7), (8) и (10) имеем

$$
\int_{0}^{\infty}\left|V_{0}^{-1}(t) R(t) \Psi(t)\right| d t<\infty
$$

Следовательно,

$$
\Psi(r)=V_{0}(r) S+\int_{r}^{\infty} V_{0}(r) V_{0}^{-1}(t) R(t) \Psi(t) d t
$$

где

$$
S=\left(\begin{array}{l}
1 \\
0
\end{array}\right)-\int_{0}^{\infty} V_{0}^{-1}(t) R(t) \Psi(t) d t .
$$

Введем угол $\beta$. Пусть $\Psi(x, r)$ - решение уравнения $(9)$, где $q$ заменено на $x q$, и пусть

$$
S(x)=\left(\begin{array}{l}
1 \\
0
\end{array}\right)-x \int_{0}^{\infty} V_{0}^{-1}(t) R(t) \Psi(x, t) d t .
$$

Очевидно, что $\Psi(x, r)$ удовлетворяет асимптотике $(10)$, а $S(x)$ - целая функция. Покажем, что $S(x) \neq 0$. Действительно, если $S\left(x_{0}\right)=0$ при некотором $x_{0} \neq 0$, то

$$
\Psi\left(x_{0}, r\right)=x_{0} \int_{r}^{\infty} V_{0}(r) V_{0}^{-1}(t) R(t) \Psi\left(x_{0}, t\right) d t .
$$

Пусть $\widetilde{\Psi}\left(x_{0}, r\right)=r^{-1 / 4} \Psi\left(x_{0}, r\right)$. Тогда

$$
\widetilde{\Psi}\left(x_{0}, r\right)=x_{0} \int_{r}^{\infty}(\sqrt{t}+1) q(t) K(r, t) \widetilde{\Psi}\left(x_{0}, t\right) d t
$$


причем матрица $K(r, t)$ в силу (8)ограничена по $t$ при каждом фиксированном $r>0$. Отсюда следует, что при выполнении условия (2) интегральный оператор в правой части последнего уравнения есть оператор Вольтерра, так что $\widetilde{\Psi}\left(x_{0}, r\right)=0, \forall r>0$, что невозможно.

Положим $\widehat{S}(x)=S(x) /|S(x)|, x \in[0,1]$. Пусть $\beta(x)$ - непрерывная функция, удовлетворяюшая следуюшим условиям:

$$
\widehat{S}(x)=\left(\begin{array}{c}
\cos \beta(x) \\
\sin \beta(x)
\end{array}\right), \quad x \in[0,1], \quad \beta(0)=0
$$

Ясно, что функция $\beta(x)$ определена однозначно. Положим $\beta=\beta(1)$.

Тогда соотношение (11) примет вид

$$
\Psi(r)=|S| V_{0}(r)\left(\begin{array}{c}
\cos \beta \\
\sin \beta
\end{array}\right)+\int_{r}^{\infty} V_{0}(r) V_{0}^{-1}(t) R(t) \Psi(t) d t .
$$

В качестве другого решения уравнения (6) возьмем функцию $\Phi(r)$, удовлетворяющую уравнению

$$
\Phi(r)=|S| V_{0}(r)\left(\begin{array}{c}
-\sin \beta \\
\cos \beta
\end{array}\right)+\int_{r}^{\infty} V_{0}(r) V_{0}^{-1}(t) R(t) \Psi(t) d t .
$$

Введя матрицу $H=(\Psi, \Phi)$, рассмотрим уравнение

$$
v(r, z)=\Psi(r)-z \int_{0}^{r} H(r) H^{-1}(t) T^{-1} J T v(t, z) d t .
$$

Лемма 2. Пусть выполнено условие (2). Тогда уравнение (14) имеет единственное решение $v(r, z)$, для которого справедливы асимптотические соотношения

$$
\begin{gathered}
v(r, z)=r^{\alpha}\left[\left(\begin{array}{l}
1 \\
0
\end{array}\right)+O(r)\right], \quad r \rightarrow 0, \quad|z| \leqslant z_{0}, \quad z_{0}-\text { постоянная, } \\
v\left(b_{z}, z\right)=|S| T_{0} M_{1}\left(b_{z}\right)\left[\left(\begin{array}{c}
-\sin \left(P_{1}\left(b_{z}\right)-\beta\right) \\
\cos \left(P_{1}\left(b_{z}\right)-\beta\right)
\end{array}\right)+O\left(z^{1-\frac{3 \delta}{2}}\right)\right], \quad z \rightarrow 0,
\end{gathered}
$$

где $b_{z}=|z|^{-\delta}, \quad 0<\delta<2 / 3$ - постоянная, не зависящая от $z$, матриць $T_{0}, M_{1}, S$ и угол $\beta$ определень соотношениями (8), (12) и (13).

ДокАЗАТЕЛЬСТво аналогично доказательству леммы 1.

Отметим, что оценка $O\left(z^{1-3 \delta / 2}\right)$ равномерна по $\arg z$.

2. Решение уравнения $(5)$ на $\left[b_{z}, \infty\right)$. Рассмотрим матрицу

$$
\begin{gathered}
D(r, z)=B^{\prime}(r, z)+B^{2}(r, z)=\operatorname{diag}\left(d_{1}, d_{2}\right), \\
d_{1,2}(r, z)=(2 m-z) z-\frac{2 A(m-z)}{r}+\frac{\alpha(\alpha \pm 1)}{r^{2}} .
\end{gathered}
$$

Пусть $a_{i}(z)$ - положительный корень уравнения $d_{i}(r, z)=0, i=1,2$. Имеем

$$
a_{i}(z)=\frac{A}{z}+O(1), \quad z \rightarrow 0
$$


Введем обозначения

$$
\begin{aligned}
\xi_{i}(r, z) & =\left(\frac{3}{2} \int_{a_{i}(z)}^{r}\left|d_{i}\right|^{\frac{1}{2}} d t\right)^{\frac{2}{3}} \operatorname{sign}\left(r-a_{i}(z)\right), \\
Q_{i}(r, z) & =\left(\xi_{i}^{\prime-\frac{1}{2}}\right)^{\prime \prime} \xi_{i}^{\prime \frac{1}{2}}, \quad Q=\operatorname{diag}\left(Q_{1}, Q_{2}\right), \\
U_{0} & =N^{\prime}-B N, \quad N=\operatorname{diag}\left(\eta_{1}, \eta_{2}\right), \\
\eta_{1} & =\xi_{1}^{\prime-\frac{1}{2}} \operatorname{Ai}\left(\xi_{1}\right), \quad \eta_{2}=\xi_{1}^{\prime-\frac{1}{2}} \operatorname{Bi}\left(\xi_{2}\right)
\end{aligned}
$$

где $\operatorname{Ai}(\xi), \operatorname{Bi}(\xi)$ - функции Эйри [3, с. 169]. Из асимптотических формул для функций Эйри вытекают следующие соотношения:

$$
U_{0}=T(z) M_{2}(r, z) \begin{cases}\left(W+O\left(P_{2}^{-1}\right)\right) \operatorname{diag}\left(\frac{1}{2} e^{-P_{2}}, e^{P_{2}}\right), & P_{2} \rightarrow+\infty \\ \widehat{U}_{0}(r, z)+O\left(P_{2}^{-1}\right), & P_{2} \rightarrow-\infty\end{cases}
$$

где

$$
\begin{gathered}
P_{2}=\int_{a_{1}(z)}^{r}\left|d_{1}\right|^{\frac{1}{2}} d t \\
T(z)=T_{0}+O(z), \quad M_{2}=\operatorname{diag}\left(\left|d_{1}\right|^{\frac{1}{4}},\left|d_{1}\right|^{-\frac{1}{4}}\right) \\
W=\left(\begin{array}{cc}
1 & 1 \\
-1 & 1
\end{array}\right), \quad \widehat{U}_{0}(r)=\left(\begin{array}{cc}
\cos \left(\pi / 4-P_{2}\right) & \sin \left(\pi / 4-P_{2}\right) \\
-\sin \left(\pi / 4-P_{2}\right) & \cos \left(\pi / 4-P_{2}\right)
\end{array}\right) .
\end{gathered}
$$

Рассмотрим уравнение

$$
u(r, z)=U_{01}+\int_{r}^{\infty} U_{0}(r, z) U_{0}^{-1}(t, z)(R(t, z)+Q(t, z)) u(t, z) d t
$$

Точно так же, как и лемма 1, доказывается следующая лемма.

ЛЕмма 3. При выполнении условия (2) решение уравнения (18) имеет следующие асимптотические разложения:

$$
\begin{gathered}
u(r, z)=\frac{A m}{2 \alpha} e^{-P_{2}} d_{1}^{-\frac{1}{4}}\left[\left(\begin{array}{c}
-1 \\
t^{2}
\end{array}\right)+o(1)\right], \quad r \rightarrow \infty, \quad z>0, \\
u\left(b_{z}, z\right)=T(z) M_{2}\left(b_{z}, z\right)\left[\left(\begin{array}{c}
\cos \left(\pi / 4-P_{2}\left(b_{z}, z\right)\right) \\
-\sin \left(\pi / 4-P_{2}\left(b_{z}, z\right)\right)
\end{array}\right)+O(I(z))\right],
\end{gathered}
$$

2дe

$$
I(z)=\int_{b_{z}}^{\infty}\left(\sqrt{t}|q(t)|+\left|d_{1}\right|^{-\frac{1}{2}}|Q(t, z)|\right) d t .
$$

4 Теоретическая и математическая физика, т. 125 , № 3,2000 г. 
3. Доказательство теоремы 1. Из утверждений лемм 2 и 3 следует, что $v(r, z) \in$ $L^{2}\left(0, b_{z}\right]$ и $u \in L^{2}\left[b_{z}, \infty\right)$, так что $\lambda$ - собственное значение задачи (3) тогда и только тогда, когда

$$
\left.\operatorname{det}(v, u)\right|_{r=b_{z}}=0 .
$$

Подставляя сюда асимптотические формулы (15) и (19), получим

$$
\cos \left(\int_{b_{z}}^{a_{1}(z)}\left|d_{1}\right|^{\frac{1}{2}} d t+\sqrt{8 A m b_{z}}-\beta-\pi \alpha-\frac{\pi}{2}\right)+o(1)=0, \quad z \rightarrow+0 .
$$

Чтобы отсюда получить асимптотику $\lambda_{n}$, рассмотрим оператор с потенциалом $V(x, r)=$ $-A / r+x q(r), x \in[0,1]$, и обозначим через $\lambda_{n}(x) n$-е собственное значение этого оператора. Тогда $\lambda_{n}$ удовлетворяет уравнению (20) при $\beta=\beta(x)$ (см. (13)). Решая это уравнение при больших $n$, будем иметь

$$
\begin{aligned}
& \left.\left(\int_{b_{z}}^{a_{1}(z)}\left|d_{1}\right|^{\frac{1}{2}} d t+\sqrt{8 A m b_{z}}-\beta-\pi \alpha-\frac{\pi}{2}\right)\right|_{z=z_{n}(x)}= \\
& \quad=(2 n-1) \frac{\pi}{2}+l_{n}(x) \pi+o(1)
\end{aligned}
$$

где $l_{n}(x)$ - целое число. Так как функция $l_{n}(x)$ непрерывна на $[0,1]$, то $l_{n}(1)=l_{n}(0)$. Но $l_{n}(0)=0$. Учитывая это, после элементарных вычислений получим

$$
\lambda_{n}=m\left(1-\frac{A^{2}}{2(n+\delta+\alpha)}\right)+o\left(n^{-1}\right), \quad n \rightarrow \infty .
$$

ЗАМЕчАНИЕ 1. Если известна дополнительная информация о скорости убывания функции $q$ на бесконечности, то можно уточнить член $o\left(n^{-1}\right)$ в $(21)$. Так, если

$$
q=O\left(r^{-\frac{3}{2}-\sigma}\right), \quad \sigma>0,
$$

то остаточный член в (19) имеет порядок $O\left(z^{\delta C}\right)$, где $C=\min \{\sigma, 1 / 2\}$. Подобрав теперь $\delta$ так, чтобы остаточные члены в формулах (15) и (19) имели одинаковый порядок, находим, что при условии (22) член $o\left(n^{-1}\right)$ в $(21)$ имеет порядок $O\left(n^{-1-\gamma}\right), \gamma=$ $2 C /(3(1+C))$.

\section{3. КВАНТОВЫЙ ДЕФЕКТ НЕПРЕРЫВНОГО СПЕКТРА}

Пусть $y(r, z)$ - решение уравнения (4), удовлетворяющее условию

$$
y(r, z) \sim r^{\alpha}\left(\begin{array}{l}
1 \\
t
\end{array}\right), \quad r \rightarrow 0,
$$

и пусть $y_{0}(r, z)$ - решение соответствуюшего невозмушенного уравнения, удовлетворяющее такому же условию. Тогда

$$
\begin{gathered}
y \sim C(\varepsilon, k) e^{\varepsilon r} r^{-\frac{A \lambda}{\varepsilon}}\left(\begin{array}{c}
m+\lambda \\
\varepsilon
\end{array}\right), r \rightarrow \infty, \\
y_{0} \sim C_{0}(\varepsilon, k) e^{\varepsilon r} r^{-\frac{A \lambda}{\varepsilon}}\left(\begin{array}{c}
m+\lambda \\
\varepsilon
\end{array}\right), \quad r \rightarrow \infty,
\end{gathered}
$$


где $\varepsilon=\sqrt{m^{2}-\lambda^{2}}, \operatorname{Re} \varepsilon>0$.

В работе [2] показано, что при выполнении условия (2) и условий 1 ) и 2$)$ (см. введение) функция $C(\varepsilon, k)$ аналитически продолжается на область $\varepsilon \notin(-\infty, 0] \cup[m,+\infty)$ и существуют конечные пределы

$$
\delta(k, \pm m)=\frac{1}{\pi} \lim _{\lambda \rightarrow \pm m \pm 0} \arg \frac{C(\varepsilon, k)}{C_{0}(\varepsilon, k)} .
$$

Мы докажем, что для сушествования пределов (25) достаточно лишь выполнения условия (2).

ЗАмечАниЕ 2. Из рассуждений работы [2] следует, что при $\lambda>m$ функция $C(\varepsilon, k)$ (соответственно $\left.C_{0}(\varepsilon, k)\right)$ совпадает с коэффициентом при слагаемом

$$
e^{-i \tau r} r \frac{A \lambda i}{\tau}\left(\begin{array}{c}
m+\lambda \\
-i \tau
\end{array}\right), \quad \tau=\sqrt{\lambda^{2}-m^{2}},
$$

в асимптотическом разложении $y(r, z)$ (соответственно $\left.y_{0}(r, z)\right)$ при $r \rightarrow \infty$.

Теорема 2. Если q удовлетворяет условию (2), то предель $\delta(k, \pm m)$ существуют и равны $\beta / \pi$, где функиия $\beta$ была определена в $n$. 1 раздела 2.

ЗАмЕчАниЕ 3. Из теоремы 2 следует, что так же, как и в регулярном случае (т.е. при выполнении условий 1) и 2)), квантовые дефекты непрерывного и дискретного спектров совпадают.

Сначала мы докажем сушествование квантового дефекта в точке $m$. Для этого построим $\Phi$ МР уравнения (5), которая в отличие от решений, введенных в [2], обладает асимптотическим разложением при $\lambda \rightarrow m+0$, равномерным по $r \in\left[b_{z}, \infty\right)$.

Лемма 4. Пусть выполнено условие (2). Тогда возмущенное и невозмущенное уравнения (5) имеют $\Phi М P U(r, z)$ и $U_{0}(r, z)$, соответственно, для которых при $\lambda \rightarrow m+0$ и $r \in\left[b_{z}, \infty\right)$ справедливы асимптотические соотношения

$$
\begin{aligned}
U(r, z), U_{0}(r, z) & \sim\left[T_{0}+O(z)\right] M_{2}[W+\Delta(r, z)] \operatorname{diag}\left(e^{-i P_{2}}, e^{i P_{2}}\right), \\
M_{2} & =\operatorname{diag}\left(|d|^{\frac{1}{4}} e^{\frac{\pi i}{4}},|d|^{-\frac{1}{4}} e^{-\frac{\pi i}{4}}\right), \\
d & =(2 m-z) z-\frac{2 A(m-z)}{r}+\frac{\alpha(\alpha+1)}{r^{2}}, \\
W & =\left(\begin{array}{cc}
-1 & 1 \\
1 & 1
\end{array}\right), \quad P_{2}=\int_{b_{z}}^{r}|d|^{\frac{1}{2}} d t \\
|\Delta(r, z)| & \leqslant C_{1}|z|+C_{2}\left(r^{-\frac{1}{2}}+\int_{r}^{\infty} \sqrt{t}|q| d t\right)
\end{aligned}
$$

постоянные $C_{1,2}$ не зависят от z. 
ДОКАЗАТЕЛЬСТВо. Положим

$$
U_{0}=N^{\prime}-B N, \quad N=\operatorname{diag}\left(\eta_{1}, \eta_{2}\right),
$$

где $\eta_{k}$ - решения уравнений

$$
\eta_{k}^{\prime \prime}-d_{k} \eta_{k}=0, \quad k=1,2
$$

Уравнения (27) имеют решения с известными ВКБ-асимптотиками на бесконечности:

$$
\eta_{k} \sim d_{k}^{-\frac{1}{4}} \exp \left((-1)^{k} i P_{2}\right)\left(1+O(r)^{-\frac{1}{2}}\right), \quad k=1,2 .
$$

Отсюда легко следует утверждение леммы.

ДОКАЗАТЕЛЬСТво ТЕОРЕМЫ 2. Пусть $v(r, z)$ - решение уравнения (5), удовлетворяюшее условию

$$
v(r, z) \sim r^{\alpha}\left(\begin{array}{l}
1 \\
0
\end{array}\right), \quad r \rightarrow 0,
$$

а $v_{0}(r, z)$ - соответствуюшее решение невозмушенного уравнения. Положим

$$
h(z)=U^{-1}\left(b_{z}, z\right) v\left(b_{z}, z\right), \quad h_{0}(z)=U^{-1}\left(b_{z}, z\right) v_{0}\left(b_{z}, z\right) .
$$

Тогда

$$
v(r, z)=U(r, z) h(z), \quad v_{0}(r, z)=U_{0}(r, z) h_{0}(z), \quad r \geqslant b_{z} .
$$

Из асимптотических формул (15) и (26) следует, что

$$
h(z) \sim \frac{|S|}{2 \sqrt{\pi}} e^{\frac{\pi i}{4}}\left(\begin{array}{c}
e^{-i \Theta} \\
e^{i \Theta}
\end{array}\right), \quad h_{0}(z) \sim \frac{|S|}{2 \sqrt{\pi}} e^{\frac{\pi i}{4}}\left(\begin{array}{c}
e^{-i \Theta_{0}} \\
e^{i \Theta_{0}}
\end{array}\right),
$$

где $\Theta=\Theta_{0}-\beta, \Theta_{0}=P_{1}\left(b_{z}\right)$.

Тогда в силу замечания $2 C(\varepsilon, k) / C_{0}(\varepsilon, k) \sim|S| e^{i \beta}$ при $\lambda \rightarrow m+0$. Отсюда и вытекает утверждение теоремы 2 для $\delta(k, m)$.

Если $\lambda<-m$, то уравнения (27) имеют точку поворота, так что в качестве решений $\eta_{k}$ следует брать эталонные решения, выражаюшиеся через функции Эйри (так же как и при вычислении квантового дефекта дискретного спектра). Все остальные рассуждения аналогичны случаю $\lambda \rightarrow m+0$.

\section{Список литературы}

[1] А. Зоммерфельд. Строение атома и спектры. Т. 1. М.: Гостехиздат, 1956.

[2] Л. А. Сахнович. ТМФ. 1996. Т. 108. № 1. С. 36-49.

[3] М.В. Федорюк. Асимптотические методы для линейных обыкновенных дифференциальных уравнений. М.: Наука, 1983. 